\section{Starch-like Polysaccharide of Streptococcus pyogenes}

MaNy strains of Lancefield group $A$ streptococci synthesize a starch-like polysaceharide when grown on a medium containing maltose or maltose-polymers ${ }^{1}$, and some of these strains also produce an extracellular amylase ${ }^{2}$. It has been shown that amylasenegative starch-accumulating strains are associated with acute glomerulonephritis and acute rheumatic fever ${ }^{3}$. There is as yet no direct proof that the starch itself is more than a metabolic marker of bacterial variants, but because of the possibility of its production in vivo its isolation and characterization were of considerable interest, for it has been shown that bacterial polysaccharides of high molecular weight may have long persistence in the tissues ${ }^{4}$.

The group $A$ strain, $I R P$ 118, type 19, was grown in 15 litres brain-heart-infusion broth, with repeated additions of maltose and automatic adjustment to $p H$. $7 \cdot 4$ during $48 \mathrm{hr}$. Thrice-washed cell suspensions (about $40 \mathrm{gm}$. dry weight) were extracted for $1 \mathrm{hr}$. at $0^{\circ}$ with $400 \mathrm{ml}$. perchloric acid. The final perchloric acid concentration used differed in different experiments, but although the yield of polysaccharide was greater with $5 \mathrm{~N}$ acid than with $2 \mathrm{~N}$ acid, the properties of the products were very similar. After clarifying by centrifugation, the extract was carefully neutralized at $0^{\circ}$ with sodium hydroxide to $p \mathbf{H} 6 \cdot 0$. On saturation of the neutralized extract with $n$-butanol and allowing to stand for two days at $0^{\circ}$, a small amount of a white precipitate appeared at the interface, but this did not stain with iodine and was therefore discarded.

The polysaccharide was then precipitated as a complex with iodine, iodine $(0.1 \mathrm{~N})$ in potassium iodide $(0 \cdot 3 \mathrm{~N})$ being added to the aqueous layer until no more blue precipitate appeared and the supernatant solution was just yellow with excess iodine. (Further addition of iodine produced a brown turbidity.) The purple precipitate was washed with dilute iodine solution and then suspended in warm distilled water and just decolorized with dilute sodium thiosulphate. The polysaccharide was then precipitated from this solution by successive additions of acetone at $0^{\circ}$, the bulk of the iodine-staining material being precipitated between 35 per cent and 45 per cent $\mathrm{v} / \mathrm{v}$ acetone. The product was washed with $n$-butanol and then with acetone and ether. The amorphous white powder thus obtained was poorly soluble in water, and solutions were best obtained by dissolving in $0.3 \mathrm{~N}$ sodium hydroxide and only neutralizing immediately before use.

It was shown by paper chromatography that the sole sugar produced by acid hydrolysis ${ }^{5}$ of the product was glucose. Reducing-sugar estimation ${ }^{6}$ after acid hydrolysis, however, showed that the product was only 95 per cent polysaccharide, the remainder being apparently inorganic salts. When calculations were based on the polysaccharide content it was found that hydrolysis with salivary $\alpha$-amylase resulted in a 100 per cent yield of maltose (as estimated by the method of Somogyi ${ }^{6}$ ) and 100 per cent hydrolysis based on iodine stain. With a commercial sample of purified $\beta$-amylase, 98 per cent maltose was liberated and the optical density of the iodine stain ${ }^{7}$ fell to 3 per cent of its original value. Since the residual iodine stain was always of the same quality as that of the original material, it seems likely that this discrepancy was due to partial retrogradation. The "blue value's of the product varied from preparation to preparation as did the yield. For example, one preparation of $60 \mathrm{mgm}$. polysaccharide from $38 \mathrm{gm}$. dry weight cells had a 'blue value' of 0.65 , and one of $200 \mathrm{mgm}$. from $49 \mathrm{gm}$. cells had a 'blue value' of $0 \cdot 8$. Controlled retrogradation of the latter product gave fractions with 'blue values' of 0.87 and 0.75 respectively and a trace of a red-staining fraction.

None of these fractions, however, showed any significant limit to $\beta$-amylolysis. It therefore seems that the polysaccharide extractable from this culture by perchloric acid is of the purely straight-chain amylose type with a range of chain-lengths from about 40 to 60 glucose units. (Based on 'blue values' for linear dextrins (Whelan, W. J., private communication).) This is confirmed by the fact that the product is completely hydrolysed when incubated in the presence of excess glucose with a preparation of amylomaltase obtained from the same strain of group $A$ streptococcus according to the method of Monod and Torriani ${ }^{9}$ for the amylomaltase of $E$. coli mutabile (ML).

Although the polysaccharide isolated does not have a very high molecular weight it exhibits a great tendency to retrograde and this retrograded form is very insoluble and might be expected to persist for long periods if it was in fact ever formed in tissues.

\section{H. BAUM}

Department of Biochemistry and Chemistry,

Royal Free Hospital School of Medicine,

8 Hunter Street,

London, W.C.1.

\section{N. Crowlex}

Department of Bacteriology,

Royal Free Hospital, Gray's Inn Road,

London, W.C.1.

${ }^{1}$ Crowley, N., and Jevons, M. P., J. Gen. Microbiol., 13, 226 (1955).

${ }^{2}$ Crowley, N., J. Gen. Microbiol., 10, 411 (1954).

${ }^{3}$ Crowley, N., J. Hyg. (Camb.), 57, 235 (1959).

"Coons, A. H., "Rheumatic Fever: A Symposium", 217 (University of Minnesota Press, 1952).

${ }^{5}$ Pirt, S. J., and Whelan, W. J., J. Sci. Food Agric., 2, 224 (1951),

-Somogyi, M., J. Biol. Chem., 160, 61 (1954).

"Bailey, J. M., and Whelan, W. J., Biochem. J., 67, 540 (1957).

Bourne, E. J., Haworth, W. N., Macey, A., and Peat, S., J. Chem. Soc., 924 (1948).

- Monod, J., and Torriani, A. M., Ann. Inst. Pasteur, 78, 65 (1950).

\section{Ubiquinone (50) and Ubichromenol in Aspergillus fumigatus, Fresenius}

Following the recent discovery ${ }^{1-5}$ of a series of ubiquinones in various biological tissues, a useful system for studying their biosynthesis was sought. A strain of Aspergillus fumigatus, Fresenius (we wish to thank Prof. J. H. Birkinshaw for the gift of a specimen culture), studied by Anslow and Raistrick ${ }^{6}$, was observed to secrete 3-hydroxy, 4-methoxy, 2,5-toluquinone (fumigatin) into the medium. This compound is closely related to the ring nucleus of ubiquinone and it seemed possible that the above organism might also produce sufficient ubiquinone for the study of its biosynthesis.

Cultures of the fungus were grown at $30^{\circ}$ in Roux bottles, each containing $200 \mathrm{ml}$. Raulin-Thom medium at $p \mathrm{H} 3 \cdot 8$. After various intervals from 3 days to 8 weeks, the thalli from groups of 2 bottles were removed and examined for ubiquinone. In the preliminary experiments, the tissue was saponified directly in methanolic potassium hydroxide in the 